\title{
Autologous Anti-MART-1 F5 T-Cell Receptor Gene-Engineered Peripheral Blood Lymphocytes
}

National Cancer Institute

\section{Source}

National Cancer Institute. Autologous Anti-MART-1 F5 T-Cell Receptor Gene-Engineered Peripheral Blood Lymphocytes. NCI Thesaurus. Code C38587.

Human autologous peripheral blood lymphocytes (PBLs) transduced with a melanoma antigen MART -1 epitope-determined T cell receptor (TCR) gene, with potential antineoplastic activity. PBLs are isolated from a melanoma patient and pulsed with a viral vector that encodes the TCR specific for an epitope of MART-1 (F5 TCR). After expansion ex vivo, the transduced autologous PBLs, expressing this specific $T C R$, are reintroduced into the patient, and bind to melanoma cells expressing the MART - 1 antigen, which may result in specific cytotoxic T-lymphocyte (CTL) killing of MART-1-expressing melanoma cells. MART-1 (melanoma antigen recognized by T cells 1 ), also known as Melan- $A$, is a melanocyte lineage-specific transmembrane protein. 